\title{
A procedure for the assessment of low frequency noise complaints ${ }^{\text {a) }}$
}

Andrew T. Moorhouse, David C. Waddington ${ }^{\text {b) }}$, \& Mags D. Adams

Acoustics Research Centre, University of Salford, Salford, M5 4WT, United Kingdom

Date of submission: 31 March 2008

Running title: Low frequency noise complaint assessment procedure

a) Aspects of this work were presented at the 12th International Meeting on Low Frequency Noise and Vibration and its Control, Ramada Plaza Hotel, Bristol UK, 18 - 20 September 2006

b) Author to whom correspondence should be addressed. Electronic mail: d.c.waddington@salford.ac.uk; Tel (+44) 1612954989 Fax (+44) 1612955208 


\begin{abstract}
The development and application of a procedure for the assessment of low frequency noise (LFN) complaints is described. The development of the assessment method included laboratory tests and field measurements, complemented with interview-based questionnaires. Environmental health departments then conducted a series of six trials with genuine 'live' LFN complaints to test the workability and usefulness of the Procedure. The Procedure includes guidance notes and a pro-forma report with step-by-step instructions. It does not provide a prescriptive indicator of nuisance but rather gives a systematic procedure to help environmental health practitioners to form their own opinion. Examples of field measurements and application of the Procedure are presented. The Procedure and examples are likely to be of particular interest to environmental health practitioners involved in the assessment of low-frequency noise complaints. [Work funded by the Department for Environment, Food and Rural Affairs (Defra), $\mathrm{UK}]$.
\end{abstract}

PACS numbers: 43.50Ba, 43.50Rq, 43.66Lj 


\section{INTRODUCTION}

Many environmental health practitioners will be familiar with complaints about low frequency noise (LFN) in the range $20-160 \mathrm{~Hz}$. The vocabulary used by complainants to describe the noise they experience is highly consistent, and invariably they describe a noise that is intense, even deafening to them while at the same time visitors to their home may hear nothing. This discrepancy between how the sufferer perceives the sound and how it is experienced by others is one of the most perplexing aspects of LFN, and can leave the sufferer feeling increasingly isolated and confused. LFN is now a recognized problem in many countries in the world, as detailed in the review by Leventhall et al. ${ }^{1}$ This does not mean that the causes of such suffering are fully understood and many cases still go unexplained. Further, these cases usually take up disproportionately more time and effort than other noise complaints. This adds to the stress on the LFN sufferers and officers concerned.

Fundamental to the problem of the assessment of LFN complaints is the question of how it may be that one person can describe a sound as loud that few others can even hear. One possible explanation that may explain some, but by no means all cases, is based on the physiology of the human hearing system for low frequencies. The perceived loudness of low frequency sounds increases rapidly with increasing acoustic energy, and so low frequency sounds just above the threshold of hearing can be perceived as loud, even uncomfortably loud. Furthermore, individual hearing thresholds vary such that people with more sensitive hearing can hear low frequency sounds that are inaudible to others.

This situation does not often arise with higher frequency sounds because their perceived loudness increases much more slowly with increased acoustic energy. A compounding factor is that 'sensitization' to low frequency sound may occur over time, leaving the sufferer more aware 
of the sound and unable to shut it out or get used to $\mathrm{it}^{2}$. This means that a short visit to a property affected by low frequency noise does not always give an adequate impression of what it is like to actually live with the sound, making evaluation even more difficult. An appreciation of these subtleties is important, because the counterintuitive nature of low frequency sound makes it difficult to base accurate judgments on personal experience.

This paper summarizes work performed recently by Moorhouse et al. ${ }^{3-5}$ to develop a Procedure for the assessment of a LFN complaint produced as part of a Defra-funded project in the UK. It was not the intention of this work to provide guidance in locating the source of a low frequency noise. Rather, the Procedure aims to help environmental health practitioners to distinguish cases where an environmental sound is responsible for a disturbance, in which case they may be able to take some action, from those where no such action is possible. However, it is usually found that the most difficult part of an assessment is in determining the existence or otherwise of a sound that correlates with the disturbance, and if this can be established then the source can usually be found.

The structure of the paper is as follows. Section II describes the development of the Procedure, involving field measurements complemented by interview-based questionnaires, and laboratory measurements comprising audiometric and subjective tests. The Procedure itself is outlined in Section III, presenting both criterion curve and the assessment method. Field trials of the Procedure by the environmental health practitioners are described in Section IV, together with a summary of their feedback regarding practical application of the method and responses of the complainants. Limitations of the Procedure and implications for environmental health practitioners and audiologists are discussed in Section V, before the Conclusions in Section VI. 


\section{DEVELOPMENT OF THE PROCEDURE}

A complementary set of field and laboratory studies was conducted in order to establish the best form for an assessment method. In the field studies, 10 independent cases of reported LFN were investigated, as well as five control cases where no complaints had been received. In addition to making physical recordings of the sounds within complainants' residences, it was necessary to obtain a significant amount of personal data about the individuals using a comprehensive one-toone semi-structured interview schedule. In the laboratory tests, a set of 'thresholds of acceptability' were established by asking 18 subjects to set the level of various low frequency sounds to a just-acceptable level for imagined day and night situations. The sounds presented consisted of a set of tones across the low frequency range, 'real' low frequency noise extracted from field test recordings, and synthesized tones with varying degrees of fluctuation. The findings from these field and laboratory studies are summarized below.

\section{A. Field measurements}

\section{Participant recruitment}

LFN sufferers were identified with the help of Environmental Health Departments in areas that had ongoing complaint cases. Having circulated letters to local government authorities throughout the UK over 40 possible cases were identified and evaluated. Through telephone discussion with the environmental health practitioners in question, a detailed description of each case was obtained. Cases where several complaints occurred in a cluster were selected in

preference over those that were isolated complaints. Environmental health practitioners and sufferers alike were generally keen to participate. Cases were selected before any acoustical measurements were made, and no cases were discarded after recordings were performed. 


\section{Field measurement methodology}

The main objective of the field measurements was to provide a database of field data for the development of a proposed criterion. Specifically this involved collecting data with which to test proposed criteria, and to provide audio recordings for use in the laboratory tests. Although the majority of environmental noise standards specify that sound measurements should be conducted outside, it is now generally agreed that low frequency noise can only meaningfully be evaluated inside dwellings ${ }^{6}$. In this series of investigations, a single microphone was positioned at a point in the room where the complainant indicated the sound was present. An unoccupied room was used for preference and recordings were usually made between $2100 \mathrm{~h}$ and $0900 \mathrm{~h}$. Subjects performed a one-on-one interview with an experienced interviewer, detail below in B.1, and were asked to complete a log sheet giving comments on how they perceived the sound at particular times. The equipment was left to monitor unmanned for between 3 and 5 days. The microphone and measurement chain were calibrated down to $1 \mathrm{~Hz}$ against a traceable standard at the UKAS accredited Calibration Laboratory at Salford University immediately prior to the tests. Parameters recorded included 1/3 octave spectra and audio. Data were streamed directly to hard disk.

\section{Analysis of the field measurements}

Large amounts of data were collected and details of the analyses are presented in the project report ${ }^{2}$. Most of the problem and marginal cases were in the 40 and $50 \mathrm{~Hz}$ bands. In all cases, the background noise levels in the residences were remarkably low. Such low levels of natural masking noise are thought to be a factor contributing to the disturbance of $\mathrm{LFN}^{1}$. Audio recordings were analysed to detect tonal components, temporal structures, and modulations that cannot be adequately detected from $1 / 3$-octave sound pressure levels alone. No such features 
were found to correlate with the complaints encounter in this study. However, audio recordings were played back at a higher level to distinguish between various noise sources. Combined with third octave and narrow band spectra, this provided the most successful identification of sources.

During field trials, there were no cases in which the LFN was reported to be present only during the day. This does not mean that the noise was absent during the day though, since most respondents said that while sound could be heard during the day, it was worst at night. Furthermore, in every case the noise was reported to be present at night. This contrasts with consultancy experience where a random selection of general industrial noise complaints might be expected to include some complaints about industry that does not operate at night that causes disturbance in the daytime. Whilst this observation does not contribute to the main method of the assessment of LFN complaints, a combination of very low background noise levels and intermittent interaction tones from domestic equipment such as refrigerators, with occasional transportation noise and room resonance modes, ought not to be overlooked when analyzing interview and measurement data.

\section{Categorization of case studies}

In the field studies, ten independent cases of reported LFN were investigated, as well as five control cases where no complaints had been received. The data from the field studies were combined with results from the laboratory test to produce a criterion curve to assist environmental health practitioners in their assessment of LFN complaints. Details of the laboratory tests are presented below in Section C, while the criterion curve is detailed in III.A. Examining the control cases, four of the five exceeded the criterion curve, the fifth being an anechoic chamber. Examining the cases where LFN was reported, three cases were identified where the criteria were exceeded and there was correlation between the residents' logged 
complaints and the low frequency noise level. Two of these three cases were related having been measured in the same apartment block. Five cases were identified where the criteria were not generally exceeded and there was a lack of correlation between comments and noise levels. Analysis of these eight cases using the Procedure for the Assessment of LFN Complaints was straightforward. However, three cases were marginal in that the measured low frequency noise was close to the criterion in level, and moreover, did not correlate with complainant comments. Investigation of these marginal cases was found to be most time-consuming. Categorization following analysis of the case studies is summarized in Table I, and examples are discussed below.

\section{Case where an environmental sound was positively identified}

This case study took place in an apartment in a quiet urban area. FIG. 1 shows a 1/3 octave band spectrum calculated over one of many periods identified by the complainant. Compared with the nighttime criterion curve from the Procedure for the Assessment of LFN Complaints, it is seen that the $63 \mathrm{~Hz} 1 / 3$ octave band predominates. FIG. 2 shows a time history of the measurements in the $63 \mathrm{~Hz} 1 / 3$ octave spectrum band, and it is evident that a source cycles on and off with periodicity of about 10 minutes on and 20 minutes off. Also shown in the time history are the $63 \mathrm{~Hz}$ daytime and nighttime criteria from the Procedure. Whilst the background level during the nighttime is well below the criterion, the source levels clearly exceed the criterion. Given the correlation of the complainant's log with these recordings these results indicate that this source is likely to be the cause of the complaints. 


\section{Case in which no environmental sound was identified}

One example of this category of case study took place in a house in a quiet urban area. Comparing the spectrum for one of the many periods identified by the complainant with the criterion curve in FIG. 3, it is seen that no particular 1/3-octave band dominates. The 63 to $100 \mathrm{~Hz}$ bands may just be audible, but the dominant source in this part of the spectrum was found to be road traffic. This was found to be quite common in the cases and control cases in this study. FIG. 4 shows a time profile of the measurements in the $80 \mathrm{~Hz} 1 / 3$ octave spectrum band. The profile of the sound levels during the night is again typical of traffic. Occasional spikes on this plot are due to domestic movement or traffic events and are not associated with any steady low frequency sound. Also shown in the time history are the $80 \mathrm{~Hz}$ daytime and nighttime criteria from the Procedure for the Assessment of LFN Complaints.

Whilst the background level during the nighttime is well below the criterion, daytime levels are also seen to be remarkably low. More detailed frequency analyses were also performed, and several other times were evaluated. However, no relationship between noise levels and the complainant's log could be established. Given the exceptionally low levels as compared with the criteria and the lack of correlation between the complainant's log with these recordings, these results indicate that no environmental source was measured that is likely to be the cause of the complaints.

\section{B. Social effects of LFN on sufferers}

\section{The qualitative methodology}

In this section, the rationale for collecting details during the fieldwork about individual's residential and occupational histories is presented. Many complainants have ongoing problems 
that they associate with low frequency noise, and which have a serious impact on their lives. However, human reaction to sound is known to be dependent not just on the sound itself, but on a complex array of other factors ${ }^{7}$. In addition to making physical recordings of the sounds within complainants' residences, it was therefore necessary to obtain a significant amount of personal data from the individuals themselves. This was important in order to obtain an overview of the background to the LFN complaint that might have a bearing on the responses.

Interviews were performed by an experienced interviewer in the complainants home, during the daytime whilst the acoustical measurement equipment was being installed. Using a comprehensive one-to-one structured interview schedule, details were collected about each individual's residential and occupational histories, their general health, details of the noise they were exposed to, suspected sources of the noise, effects of the noise on themselves and their health, and any measures they have taken to cope with or avoid the noise. Each participant of the field trials answered all questions without hesitation, and were forthcoming and open when answering questions relating to their general and mental health, and when providing detailed information about their noise problem.

\section{Symptoms reported by LFN complainants}

Reactions to the problem ranged from an annoyed interest to feeling suicidal. Symptoms were identified by asking complainants a number of personal questions about their current general health. Firstly, they were asked to describe their general health in their own words. They were then asked to list any symptoms they suffered from, whether or not they attributed these to the noise problem, and to indicate for how long they had suffered each symptom. They were asked about any known hearing problems, when they had last had a hearing test, what the outcome of the test was, and whether they were satisfied with that outcome. Each complainant was 
specifically asked if they suffered from tinnitus, although they were not examined by an otolaryngologist. This means that their self-reporting may have been inaccurate. Following this detailed health discussion, which allowed complainants to name their health problems in their own words, a final question was asked where a list of other symptoms was read out and the complainant was asked whether they suffered from any of them. The list of symptoms was based on that published by Leventhall ${ }^{1}$. Again, it was made clear that they should say whether they suffered from the symptom whether or not they attributed it to exposure to LFN. The combination of open questions and the list of known symptoms meant that a full set of health issues were identified for each complainant. Some complainants practiced successful coping strategies at the time of the interview and so were asked to report health problems at the time when their suffering from the noise had been at its worst. Table II summarizes some of the more striking findings.

\section{Discussion of findings from the semi-structured interview}

The results indicate that all the complainants used in the study had ongoing problems that they associated with low frequency noise, and that had a serious impact on their quality of life. None of the complainants had a history of suffering from these problems at previous residences, and none had an employment or other discernable relationship with the company or organization suspected as the source of the low frequency noise about which they complained. Furthermore, as far as can be judged by an experienced interviewer, the complaints were genuine, and there was no hint of ulterior motives. 


\section{Assessment of methodology}

Combining measurements with a questionnaire gave a significant amount of personal data about the individuals and gave an overview of the background to the LFN complaint that might have a bearing on the responses. These sociological factors were incorporated into the Procedure in the form of a questionnaire to be used by the investigating environmental health practitioners. The answers to the questions help local authorities distinguish cases where they should intervene from those where they can do nothing to help.

\section{Laboratory tests}

\section{Objectives of the laboratory tests}

The objective of the laboratory tests was to establish 'thresholds of acceptability' for lowfrequency sounds, for day and night exposure. Previous work, including most national guidelines ${ }^{1}$, is based on the idea that the acceptability of a low frequency sound can be evaluated in relation to a frequency-dependent reference curve. Such a curve can be called the 'threshold of acceptability': sounds with a higher intensity would be considered unacceptable, and those with a lower intensity acceptable.

A further objective of these tests was to investigate the effect of fluctuations on the disturbance caused by low frequency noise. Specifically the questions to be addressed were:

i. Should fluctuating low frequency sounds be penalized compared with steady sounds?

ii. If so, then by how much?

iii. What measured parameter(s) should be used to determine when such a penalty should be applied? 
The aim was to derive a method suitable for use by environmental health practitioners to quantify the effect of fluctuations. It is not possible to reproduce realistic field conditions in a laboratory test. In particular, the length of exposure does not give an adequate impression of what it is like to live with the sound. Therefore, it was not the objective of these laboratory tests to establish absolute levels for a reference curve.

\section{Methodology for laboratory tests}

The 'threshold of acceptability' is defined as the level of a particular sound that the subject judges to be just acceptable for an assumed daytime or nighttime situation. Thresholds of acceptability were determined by the method of adjustment for a number of fluctuating and steady sounds. The subject was seated in a simulated living room into which pre-recorded lowfrequency sounds were to be played, and the following instructions were read to the subject.

"Imagine you are at home during the day. Press the button whenever you consider the sound is not acceptable to live with and keep it pressed. Whenever you consider the sound is acceptable to live with, release the button."

An operator then adjusted levels using similar techniques to those used in audiometry, reducing the level of the sound gradually when the button was pressed until it was released. Each sample lasted 90 seconds, which had been found during preliminary tests to be sufficient time to obtain a reliable threshold. It was found that after an initial training period the threshold levels were repeatedly set to within $1 \mathrm{~dB}$. For the 'nighttime' tests the main lights were switched off and the first sentence of the instruction was replaced by: "Imagine you are at home at night and trying to get to sleep." 
The set of sounds presented to subjects comprised a combination of real and synthesized sounds that was developed and refined during a series of preliminary tests. Three sets of sounds were used:
a. Real sounds from field recordings
b. Steady synthesized tones
c. Beating synthesized tones.

The advantage of real sounds is that they are known to have caused disturbance. The advantage of synthesized sounds is that they can be controlled so that only one aspect of the sound is varied at once. Specifically, this allowed control of the amount of fluctuation whilst keeping other characteristics of the sound constant. The real sounds were taken from the field measurements made in the dwellings of LFN sufferers. It was necessary to ensure that parameters such as tonality and frequency content were kept constant, and that only the fluctuation varied. Synthesized tones were constructed from $40 \mathrm{~Hz}$ and $60 \mathrm{~Hz}$ sinusoids, with steady tones of single sinusoids, and of two sinusoids of similar frequencies as shown in Table III.

\section{Choice of subjects}

The choice of both the number and make up of subjects was an important consideration. Regarding the profile of subjects, LFN sufferers tend to be middle aged or elderly, and the majority is women ${ }^{1}$. In addition, there is evidence that people known to be disturbed by LFN will judge sounds differently to a cross section of non-sufferers. Consequently, the profile summarized in Table IV was chosen. 


\section{Low frequency hearing thresholds}

A conventional audiometric test was conducted on each subject over the frequency range $250 \mathrm{~Hz}-$ $6 \mathrm{kHz}$ to identify any hearing defects that could affect the results. In addition, low frequency audiometric tests were carried out in an anechoic chamber using pure tones played through a loudspeaker at the third octave band center frequencies between 31.5 and $160 \mathrm{~Hz}$. Each subject took part in three listening sessions and one training session, each lasting 20 minutes.

FIG. 5 shows the hearing thresholds of all subjects averaged over each group. There was a spread of between 25 and $40 \mathrm{~dB}$ between the most and least sensitive subjects. The younger age group (group 2) has more sensitive hearing than the 55-70 year old group (group 1) by about $5 \mathrm{~dB}$ as

might be expected. The shapes of the spectra follow the ISO reference threshold of hearing, and the levels show good agreement given that the ISO curve applies to 18-25 year olds whereas the average age of the subjects was 60 and 32 years for group 1 and 2 respectively. The least sensitive group in terms of hearing threshold is group 0 (sufferers).

\section{Threshold of acceptability for real sounds}

Thresholds of acceptability for the real sounds in the nighttime are shown in FIG. 6 for all subjects. There is a wide spread of results which might be expected given the wide range of hearing thresholds. However, the lines are surprisingly parallel, indicating that all subjects responded in a similar way to the various sounds, but at a different overall level.

FIG. 7 shows the same data as FIG. 6 but averaged by group. We see that group 0 (sufferers) is less sensitive in absolute terms than the other groups, by about 2 to $4 \mathrm{~dB}$. There is no significant difference in the responses of the other two groups. Subjects were generally more tolerant of 
track 1, which displayed the smallest fluctuations by about $5 \mathrm{~dB}$, and judged the other four sounds to be similar in terms of their acceptability.

It might be expected that acceptability thresholds would depend on hearing thresholds, and it is therefore interesting to examine the difference between these two thresholds for each subject. These data are given in FIG. 8 for the nighttime scenario. On average respondents set the nighttime thresholds $2 \mathrm{~dB}$ lower than for the day, and the difference between day and night was almost identical for each sound. This result suggests that there was no qualitative difference in the sounds, and that no particular sound was relatively more disturbing just at night. Two important points can be derived from these data.

i. sufferers tend to set acceptable levels close to their threshold of hearing, both day and night

ii. the youngest group was most tolerant, and the older group less so, to these sounds.

In absolute terms, the sufferers in these tests were the least sensitive group to low frequency sounds. A major factor in this is that sufferers' thresholds of hearing were higher than those of other groups. We should avoid strong general conclusions because only three sufferers were tested, and there was variation between them. Nevertheless, this finding contradicts the view sometimes expressed that LFN problems are a result of exceptional sensitivity. In relative terms, sufferers tend to set the threshold of acceptability much closer to the threshold of hearing than other groups. Whether this is because they are naturally less tolerant, or have become sensitized by exposure is not known. 


\section{Threshold of acceptability for steady tones}

FIG. 9 shows the thresholds of acceptability for steady tones in the nighttime scenario set by all subjects averaged over each group. There was a spread of about $30 \mathrm{~dB}$ between the most and least sensitive subject. This is not surprising given that the thresholds of hearing have a similar spread. In absolute terms, the LFN sufferers are the least sensitive group, followed by the older and then the younger group. As mentioned above, this contradicts the often-held view that LFN sufferers tend to be particularly sensitive.

Shown in FIG. 10 are the relative nighttime acceptability thresholds, i.e. the difference between the threshold of acceptability and of hearing for each individual, averaged by group. There was $\sim 35 \mathrm{~dB}$ spread in the results. Some subjects set the threshold of acceptability only a few $\mathrm{dB}$ above their hearing threshold, judging that a sound that was only just audible to be unacceptable. Others set the difference very much higher, so that the sound would be clearly audible before they judged it unacceptable.

Two points of interest can be made. Firstly, there is a marked difference in the average response of LFN sufferers compared with the other two groups. LFN sufferers set the acceptable level about $10 \mathrm{~dB}$ higher than hearing threshold on average, whereas for non-sufferers, the difference was about $20 \mathrm{~dB}$. Thus, we can say that relative to their hearing threshold the LFN sufferers are more sensitive than are non-sufferers, although as stated above in absolute terms they were less sensitive. However, we should again be cautious about drawing general conclusions based on three subjects.

The second point is that for the lower frequency bands, the threshold of acceptability reduces, i.e. gets closer to the threshold of hearing. This is significant since it suggests that the optimum 
shape of a reference curve does not follow the threshold of audibility over the whole of the low frequency range. Rather, it will tend to follow the hearing threshold for the lower bands but then move away from it above around $50 \mathrm{~Hz}$.

\section{Threshold of acceptability for beating tones}

Referring to FIG. 11, there are several clear trends. Firstly, as before, Group 0 (LFN sufferers) is the most sensitive group in relative terms, setting the acceptability threshold only $2-3 \mathrm{~dB}$ above audibility threshold for nighttime beating tones. Secondly, subjects were 3-5dB more tolerant of steady tones than of the corresponding beating tone. This is consistent with previous published research ${ }^{9}$, and proposed revisions to American National Standard criteria for evaluating room noise with regard to quiet HVAC system design ${ }^{10,11}$. This is also consistent with the Danish standard $^{12}$ method of adding a $5 \mathrm{~dB}$ penalty for impulsive noise, as well as existing UK guidelines ${ }^{13}$ where a $5 \mathrm{~dB}$ penalty is added for noise with noticeable features.

Thirdly, daytime levels were set an average of $3-4 \mathrm{~dB}$ higher than the corresponding nighttime levels. This is a slightly lower difference than the $5 \mathrm{~dB}$ daytime relaxation used in the German standard ${ }^{14}$. However, due to difficulty in reproducing realistic night-time conditions, it is likely that this difference is underestimated in the laboratory tests ${ }^{15}$. Consequently, $5 \mathrm{~dB}$ is an appropriate relaxation to the limits for sounds only present during the day. Lastly, the effect of the beating on the response was essentially the same for day and night. This means that the procedure used to assess fluctuations can be applied equally to night and day. These last two points are illustrated most clearly in FIG. 11.

Two alternative methods are suggested here for the assessment of a sound for fluctuation. The first is based on the parameter known as prominence ${ }^{16}$, and is that a sound should only be 
considered fluctuating when the rate of change of the RMS Fast sound level in the third octave band of interest exceeds $10 \mathrm{~dB}$ per second. The second method uses the difference $\mathrm{L}_{10}-\mathrm{L}_{90}$, which has the additional advantage that it is generally available to environmental health practitioners. Shown in FIG. 12 are the relative nighttime acceptability plotted against the value of $\mathrm{L}_{10}-\mathrm{L}_{90}$ for each sound averaged for all subjects. In one of the preliminary tests, subjects were played a sequence of beating tones with varying degrees of fluctuation. It was found that the relative thresholds of acceptability were set at about the same level for the various beating tones, but that there was a clear difference of $\sim 5 \mathrm{~dB}$ from those for the steady tones.

Arguably, FIG. 12 also displays this trend: the most fluctuating sounds, represented by points to the right, display a 'penalty' of $\sim 5 \mathrm{~dB}$ compared with steady sounds on the left. The overall trend can be simplified without much loss of accuracy by ignoring the short transition range. The simplified trend can then be described as follows:

i. $\mathrm{L}_{10}-\mathrm{L}_{90}<5 \mathrm{~dB}: \quad$ no penalty

ii. $\mathrm{L}_{10}-\mathrm{L}_{90}>5 \mathrm{~dB}$ : penalty of $5 \mathrm{~dB}$.

This is in a form that could be used by environmental health practitioners to decide whether to apply the $5 \mathrm{~dB}$ penalty. Although useful, the difference $\mathrm{L}_{10}-\mathrm{L}_{90}$ is not a foolproof parameter since it does not include any effect of the rate of fluctuations.

\section{LFN ASSESSMENT PROCEDURE}

The LFN assessment procedure is detailed in the Procedure for the Assessment of LFN Complaints ${ }^{5}$, together with guidance notes and a pro-forma report with systematic instructions. Measurements for the Procedure for the Assessment of LFN Complaints require detailed 
acoustical monitoring over a period of three to five days combined with a synchronized log completed by the complainant. There are then two aspects to the assessment procedure:

1. Comparison of the level of recorded sound with a third octave band criterion curve

2. Evaluation of the correlation between the recorded sound and the complainant's log.

\section{A. The criterion curve}

The criterion curve is given in Table V and FIG 13. If the noise occurs only during the day then $5 \mathrm{~dB}$ relaxation may be applied to all third octave bands. Note that the criterion curve sound levels given in Table $\mathrm{V}$ for $25 \mathrm{~Hz}$ and below can cause the vibration of windows, walls and even floors in residential housing structures with the accompanying rattling of dishes and bric-a-brac. This induced vibration and the accompanying secondary noises will be noticed by residents, with annoyance the likely result. Some account of vibration-induced noise is made in the Japanese method for the assessment of low-frequency noise complaints ${ }^{17}$.

\section{B. Evaluation of the recordings and complainant's log}

The following provides a step-by-step guide to analysis.

1. Consult the complainant's log to find times when the sound was considered most disturbing.

2. If possible, check the character of the sound at these times by audio playback.

3. If the sound is predominantly due to traffic or movement within the building then reject this sample.

4. For the chosen time obtain the third octave band spectrum of $\mathrm{L}_{\mathrm{eq}, \mathrm{T}}$ samples 
5. Compare the $\mathrm{L}_{\mathrm{eq}, \mathrm{T}}$ spectrum to the criterion curve to find any third octave bands for which the criterion curve is exceeded.

6. For the third octave band which exceeds the curve by the greatest margin plot the time variation of the $\mathrm{L}_{\mathrm{eq}, \mathrm{T}}$ for the 24 hour period in which the event occurred.

7. Compare the complainant's log with the time history to see whether there is correlation between the two.

\section{FIELD TRIALS BY ENVIRONMENTAL HEALTH PRACTITIONERS}

\section{A. Objectives of the field trials}

Generally, some caution is needed in applying laboratory test results to real situations since laboratory experiments cannot reproduce the possible effects of sensitization over time, or account for the physical modification and enhancement of the experienced sound field in-situ. Nevertheless, it is believed that findings from laboratory testing can be reliably applied to provide a clearer understanding of the disturbance experienced by LFN sufferers in their homes. Consequently, it was resolved to undertake genuine trials of the Procedure. Cases were solicited by letter and email requests to 62 local authorities around the UK, and a series of six trials of 'live’ LFN complaints was conducted by volunteers from Environmental Health departments.

\section{B. Results of the field trials}

In two out of the six cases an environmental noise was identified and its source located. In the remaining four cases, no environmental noise was found and the officers concluded there was no remedial action they could take. In each field study, the sound measurements were supported by a semi-structured interview as detailed in the Procedure, to determine whether sociological or

other factors might influence the results. Combining measurements with a questionnaire 
provided a significant amount of personal data about the individuals and an overview of the background to each LFN complaint that might have a bearing on the responses to the perceived noise.

\section{Findings from the debriefing session}

\section{General comments}

A debriefing session was arranged to obtain feedback from the environmental health practitioners following application of the Procedure to their 'live' cases. The environmental health practitioners' experience in applying the Procedure was generally very positive: the participating officers found the Procedure easy to use and that working to a set procedure increased their confidence and the complainant's acceptance of the results. They also considered that the Procedure achieved a good balance, giving a set method but allowing them the flexibility to form their own conclusions.

\section{Experience of the semi-structured interview}

With particular reference to the environmental health practitioners' evaluation of the subjective part of the Procedure, it was felt that the interview provided a formal way of acquiring sensitive information that was relevant to the analysis. During the debriefing one practitioner confirmed, "[The complainant was] generally happy to be asked. It showed we were leaving no stone unturned." It also engendered trust and confidence in the environmental health practitioner on the part of the complainant who could see the rigor that was being applied to their case. The interviews gave the complainants the sense that they were being listened to and that everything possible was being done to help them. In particular, it was recognized; "Doing an interview that is formalized makes us able to tell them this is a way of gathering data and provides us with a 
pathway to give us confidence. It meant the complainant could see we'd done our best." Subsequently, some complainants in the field trials were satisfied that their case was closed even though no environmental source was found.

\section{Assessment of application of the Procedure in the field}

The environmental health practitioners were generally able to draw firm conclusions and reach 'closure' even if there was nothing they could do to help. As one Practitioner stated, "The Procedure... raised our credibility and the complainant's acceptance of the findings". The environmental health practitioners who had found no low frequency environmental noise present commented that the lack of an alternative, more appropriate course of action for the complainants was a remaining difficulty. Currently there is no further formal advice that can be given to help complainants who are still suffering with their problem. There was a strong feeling that officers need somewhere to send people affected in this way. Consequently, there was a sentiment amongst the environmental health practitioners that an initiative to develop a further course of action would be endorsed, perhaps along the lines of 'relief strategies'.

\section{DISCUSSION}

In many cases, environmental health practitioners will find a noise source above audible thresholds that clearly correlates with the complainant's log, typically an industrial process of some kind, fans, pumps or electrical equipment. However, in eight of the eleven cases considered in the fieldwork, no environmental source could be found for the LFN complaint. In fact, a striking feature about many LFN sufferers' homes considered was the almost complete absence of any intrusive environmental noise. Further, in four out of the six field trials of the Procedure 
performed by Environmental Health officers, no environmental noise consistent with the complaint could be found.

The proposed criteria curve is provided as guidance for environmental health officers in their evaluation of an LFN complaint, and not as an absolute limit. This means that tonal sounds at, or just below, the threshold of the hearing should be considered as environmental sources potentially responsible for the complaint. The course of action when no environmental noise consistent with the complaint can be found, and yet the complainant is clear distressed, is unclear. On one hand, suggestion that a medical screening for tinnitus is in order is often irately rejected by the complainant. On the other hand, the environmental health officer could simply close the case and avoid further involvement as it may lead to frustration and false hopes. However, this strategy is unacceptable to many environmental health practitioners since it involves leaving a problem unsolved with the complainant still in distress.

An answer to this conundrum may lie in the existing clinical audiology and auditory neuroscience literature. A complement to the Procedure would be the development of techniques by which the sufferer might acquire a degree of control over their adverse reactions. Applying neuropsychological understanding of human hearing and tinnitus to LFN complaints, Moorhouse and Baguley ${ }^{18}$ are proposing that environmental health officers who have applied the Procedure and not identified an environmental sound that could account for the complaint could refer cases to strategically located Audiology Departments. A similar approach has been trialed in Japan by Kitamura et al. ${ }^{17}$. A caveat to this approach however is that the LFN sufferer has to understand that no one can simply turn the sound off: it is up to him or her to solve this problem, as is also the case with tinnitus. Such a network could be established by providing specialist audiologists with some additional background knowledge about LFN. 


\section{CONCLUSIONS}

Until recently, it has been extremely difficult for environmental health practitioners in the UK and elsewhere to deal with complaints about LFN. This was in part because no official guidance was available to support them. The UK Defra Procedure for the assessment of low frequency noise complaints has addressed this point. Feedback from environmental health practitioners taking part in field trials of the Procedure has been very positive, indicating that Procedure was easy to follow and strengthened the authority's position with the complainant. Furthermore, complainants were said to be significantly reassured once they saw that a detailed procedure was being followed. We expect a reasonable proportion of cases to remain unresolved even with the application of the Procedure, since a 'no environmental source found' conclusion may not resolve the matter for many LFN sufferers. Nevertheless, this does not negate the value of a Procedure that provides environmental health practitioners with a means of distinguishing cases where they should act from those where they can do nothing to help. It does however indicate the need for some alternative for those LFN sufferers not satisfied with the outcome.

In absolute terms, the sufferers that participated in the laboratory tests were group least sensitive to low frequency sounds. A significant factor is that their thresholds of hearing were higher than other groups. This finding contradicts the view sometimes expressed that LFN problems are a result of exceptional sensitivity. Thresholds of acceptability were set typically 3-5dB higher for sounds with strong fluctuations than for steady sounds. It is therefore appropriate to penalize fluctuating sounds compared with steady sounds, and that $5 \mathrm{~dB}$ is an appropriate level for such a fluctuation penalty. Although the laboratory tests yielded some interesting results, strong conclusions cannot be drawn due to the small sample size. 


\section{ACKNOWLEDGEMENTS}

The authors are grateful to Defra for funding the work leading to this paper. Thanks are due to Dr. Geoff Leventhall, and we would like to acknowledge the contribution of Dr David Baguley. We would like to thank the environmental health practitioners that performed the field trials of the Procedure. The authors would like to thank the referees for their helpful comments. 


\section{REFERENCES}

${ }^{1}$ G. Leventhall, P. Pelmear, and S. Benton, "A Review of Published Research on Low Frequency Noise and its Effects," Department for Environment, Food and Rural Affairs, London, 2003. http://www.defra.gov.uk/environment/noise/research/lowfrequency/pdf/lowfreqnoise.pdf Last viewed 31 March 2008

${ }^{2}$ H. Guest, "Inadequate standards currently applied by local authorities to determine statutory nuisance from LF and infrasound," Journal of Low Frequency Noise, Vibration and Active Control, vol. 22, pp. 1-7, 2003.

${ }^{3}$ A. T. Moorhouse, D. C. Waddington, and M. Adams, "Proposed criteria for the assessment of low frequency noise disturbance," Department for Environment, Food and Rural Affairs, London, 2004.

http://www.defra.gov.uk/environment/noise/research/lowfrequency/pdf/nanr45-criteria.pdf $\quad$ Last viewed 31 March 2008

${ }^{4}$ A. T. Moorhouse, D. C. Waddington, and M. Adams, "Field trials of proposed procedure for the assessment of low frequency noise complaints," Department for Environment, Food and Rural Affairs, London, 2004.

http://www.defra.gov.uk/environment/noise/research/lowfrequency/pdf/nanr45-fieldtrials.pdf Last viewed 31 March 2008

${ }^{5}$ A. T. Moorhouse, D. C. Waddington, and M. Adams, "Procedure for the assessment of low frequency noise disturbance," Department for Environment, Food and Rural Affairs, London, 2005.

http://www.defra.gov.uk/environment/noise/research/lowfrequency/pdf/nanr45-procedure.pdf Last viewed 31 March 2008 
${ }^{5}$ BS ISO 226:2003 : Acoustics. Normal equal-loudness-level contours: British Standards Publishing Limited.

${ }^{6} \mathrm{P}$. Schomer, "The importance of proper integration of and emphasis on the low-frequency sound energies for environmental noise assessment," Noise Control Engineering Journal, vol. 52, pp. 26-39, 2004.

${ }^{7}$ B. Schulte-Fortkamp and A. Fiebig, "Soundscape Analysis in a Residential Area: An Evaluation of Noise and People's Mind," Acta Acustica united with Acustica, vol. 92, pp. 875-880, 2006.

${ }^{8}$ J. S. Bradley, "Annoyance Caused by Constant-Amplitude and Amplitude-Modulated Sounds Containing Rumble," Noise Control Engineering Journal 42 (6), 203 (1994)..

${ }^{9}$ T. Poulsen and F. R. Mortensen, "Laboratory Evaluation of Annoyance of Low Frequency Noise," Danish Environmental Protection Agency 2002.

${ }^{10}$ P. D. Schomer, "Proposed revisions to room noise criteria," Noise Control Engineering Journal, vol. 48, pp. 85-96, 2000.

${ }^{11}$ P. D. Schomer and J. S. Bradley, "A test of proposed revisions to room noise criteria curves," Noise Control Engineering Journal, vol. 48, pp. 124-129, 2000.

${ }^{12} \mathrm{~J}$. Jakobsen, "Danish guidlines on environmental low frequency noise, infrasound and vibration," Jnl Low Freq Noise Vibn \& Active Control, vol. 20, pp. 141-148, 2001.

${ }^{13} \mathrm{BS}$ 4142:1997 : Method for rating industrial noise affecting mixed residential and industrial areas: British Standards Limited.

${ }^{14}$ Standard DIN 45680, "Measurement and rating of low frequency noise emission in the neighbourhood, (in German)," Deutsches Institut für Normung e.V., Berlin. 1997. 
${ }^{15}$ Y. Inukai, N. Nakamura, and H. Taya, "Unpleasantness and acceptable limits of low frequency sound," Journal of Low Frequency Noise, Vibration and Active Control 19, 135 (2000).

${ }^{16}$ T. H. Pederson, "Objective method for measuring the prominence of impulsive sounds and for adjustment of LAeq," presented at InterNoise, The Hague, The Netherlands, 2001.

${ }^{17}$ T. Kitamura, M. Hasebe, and S. Yamada, "Psychological analysis of complainants on noise/low frequency noise and the relation between psychological response and brain structure," Journal of Low Frequency Noise Vibration and Active Control 24 (1), 43 (2005).

${ }^{18}$ A. T. Moorhouse and D. Baguley, "Sound advice: solution to the often intractable problem of low frequency noise complaints," in Environmental Health Practitioner, (2007), pp. 20. 
TABLES 
TABLE I: Categorization of case studies

\begin{tabular}{|l|c|}
\hline Environmental sound category & Number of cases \\
\hline Positively identified & 3 \\
\hline Marginal & 3 \\
\hline No environmental source found & 5 \\
\hline
\end{tabular}


TABLE II: Numbers of LFN complainants reporting selected symptoms.

\begin{tabular}{|l|c|c|}
\hline \multicolumn{1}{|c|}{ Health Issue } & Number of respondents & Percentage of respondents \\
\hline Sleep disturbance & 11 & $92 \%$ \\
\hline Stress & 10 & $83 \%$ \\
\hline Frustration & 9 & $75 \%$ \\
\hline Difficulty falling asleep & 8 & $67 \%$ \\
\hline Anxiety & 8 & $67 \%$ \\
\hline Tiredness & 7 & $58 \%$ \\
\hline Pressure or pain in ear or body & 7 & $58 \%$ \\
\hline Headaches & 7 & $58 \%$ \\
\hline Body vibration or pain & 6 & $50 \%$ \\
\hline Frequent irritation & 5 & $42 \%$ \\
\hline Insomnia & 5 & $42 \%$ \\
\hline Depression & 4 & $33 \%$ \\
\hline Migraine & 3 & $25 \%$ \\
\hline Abdominal symptoms & 3 & $25 \%$ \\
\hline Chronic fatigue & 2 & $17 \%$ \\
\hline Suicidal & 2 & $17 \%$ \\
\hline Tinnitus & $1^{\mathrm{a}}$ & \\
\hline
\end{tabular}

a) Respondent attributed whistling in ear to sinusitis rather than tinnitus 
TABLE III: Details of composition of synthesized tones

\begin{tabular}{|l|c|}
\hline Synthesized tone & Component sinusoids \\
\hline Steady 1 & $40 \mathrm{~Hz}$ at $0 \mathrm{~dB}$ \\
\hline Steady 2 & $60 \mathrm{~Hz}$ at $0 \mathrm{~dB}$ \\
\hline Beating 1 & $\begin{array}{c}40 \mathrm{~Hz} \text { at } 0 \mathrm{~dB} \\
41.5 \mathrm{~Hz} \text { at }-8 \mathrm{~dB}\end{array}$ \\
\hline Beating 2 & $60 \mathrm{~Hz}$ at $0 \mathrm{~dB}$ \\
& $61.5 \mathrm{~Hz}$ at $-8 \mathrm{~dB}$ \\
\hline
\end{tabular}


TABLE IV: Make up of subject groups for laboratory tests

\begin{tabular}{|c|l|c|c|c|c|}
\hline Group & \multicolumn{1}{|c|}{ Group profile } & $\begin{array}{c}\text { Average } \\
\text { age }\end{array}$ & Male & Female & Total \\
\hline 0 & $\begin{array}{l}\text { Subjects known to be disturbed by low frequency } \\
\text { sounds }\end{array}$ & 62 & 0 & 3 & 3 \\
\hline 1 & $\begin{array}{l}\text { Subjects with the age profile of typical LFN } \\
\text { sufferers (55-70 year old) but without a history of } \\
\text { disturbance by LFN }\end{array}$ & 60 & 5 & 3 & 8 \\
\hline 2 & $\begin{array}{l}\text { Subjects from a younger age group chosen at } \\
\text { random. }\end{array}$ & 32 & 2 & 5 & 7 \\
\hline All & \multicolumn{1}{|c|}{50} & 7 & 11 & 18 \\
\hline
\end{tabular}


TABLE V: Proposed nighttime reference curve

\begin{tabular}{|c|c|c|c|c|c|c|c|c|c|c|c|c|c|}
\hline $\mathrm{Hz}$ & 10 & $\mathbf{1 2 . 5}$ & $\mathbf{1 6}$ & $\mathbf{2 0}$ & $\mathbf{2 5}$ & $\mathbf{3 1 . 5}$ & $\mathbf{4 0}$ & $\mathbf{5 0}$ & $\mathbf{6 3}$ & $\mathbf{8 0}$ & $\mathbf{1 0 0}$ & $\mathbf{1 2 5}$ & $\mathbf{1 6 0}$ \\
\hline $\mathrm{dB}$, Leq & 92 & 87 & 83 & 74 & 64 & 56 & 49 & 43 & 42 & 40 & 38 & 36 & 34 \\
\hline
\end{tabular}




\section{COLLECTED FIGURE CAPTIONS}

FIG. 1: Case where an environmental sound was positively identified. Solid line shows measured $1 / 3$ octave band spectrum averaged over $9 \mathrm{~m} 30$ s starting $0700 \mathrm{~h}$. Dashed $(\mathrm{X})$ line is the nighttime criteria from the Procedure for the assessment of LFN complaints.

FIG. 2: Case where an environmental sound was positively identified. Time history showing

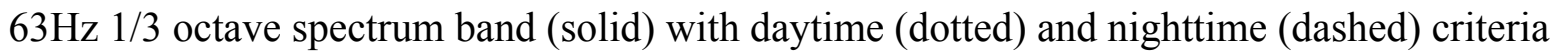

FIG. 3: Case where no environmental sound was identified. Solid line shows measured $1 / 3$ octave band spectrum averaged over 9m30s starting 1930h. Dashed (X) line is the nighttime criteria from the Procedure for the assessment of LFN complaints.

FIG. 4: Case where no environmental sound was identified. Time history showing $80 \mathrm{~Hz} 1 / 3$ octave spectrum band (solid) with daytime (dotted) and nighttime (dashed) criteria

FIG. 5: Average low frequency hearing thresholds for each group. Group 0 (LFN sufferers)(O), group 1 (55-70 years old)( ), group 2 (younger age)( $\diamond)$, ISO $226(0 \mathrm{~dB}$ threshold curve)(X)

FIG. 6 Nighttime thresholds of acceptability to real sounds, all subjects

FIG. 7 Nighttime thresholds of acceptability relative to hearing threshold for real sounds, by group. Group 0 (LFN sufferers)(O), group 1 (55-70 years old)( ), group 2 (younger age)( ()

FIG. 8 Nighttime thresholds of acceptability relative to hearing threshold for real sounds, all subjects. Group 0 (LFN sufferers)(O), group 1 (55-70 years old)( ), group 2 (younger age $)(\diamond)$

FIG. 9: Nighttime acceptability thresholds for tones by group. Group 0 (LFN sufferers)(O), group 1 (55-70 years old)( ), group 2 (younger age)( ()

FIG. 10: Nighttime acceptability thresholds for tones relative to hearing thresholds by group. Group 0 (LFN sufferers)(O), group 1 (55-70 years old)( ), group 2 (younger age)( () 
FIG. 11: Comparison of daytime $(+)$ and nighttime $(\mathrm{X})$ acceptability thresholds relative to hearing threshold for steady and beating tones. Average of all subjects.

FIG. 12 Nighttime thresholds of acceptability relative to hearing threshold for real sounds ( $\bullet$ ), steady tones $(\bullet), 40 \mathrm{~Hz}$ beating tones $(\bullet)$ and $60 \mathrm{~Hz}$ beat tones ( $\bullet$ ) averaged for all subjects. Showing variation with $\mathrm{L}_{10}-\mathrm{L}_{90}$. The beating tones datum points $(\bullet)$ and $(\triangleleft)$. are so close as to be indistinguishable. These listening test results are used to support the suggestion that a $5 \mathrm{~dB}$ penalty for fluctuations is appropriate when $\mathrm{L}_{10}-\mathrm{L}_{90}>5 \mathrm{~dB}$.

FIG. 13 Criterion curve from the Procedure for the assessment of low frequency noise 


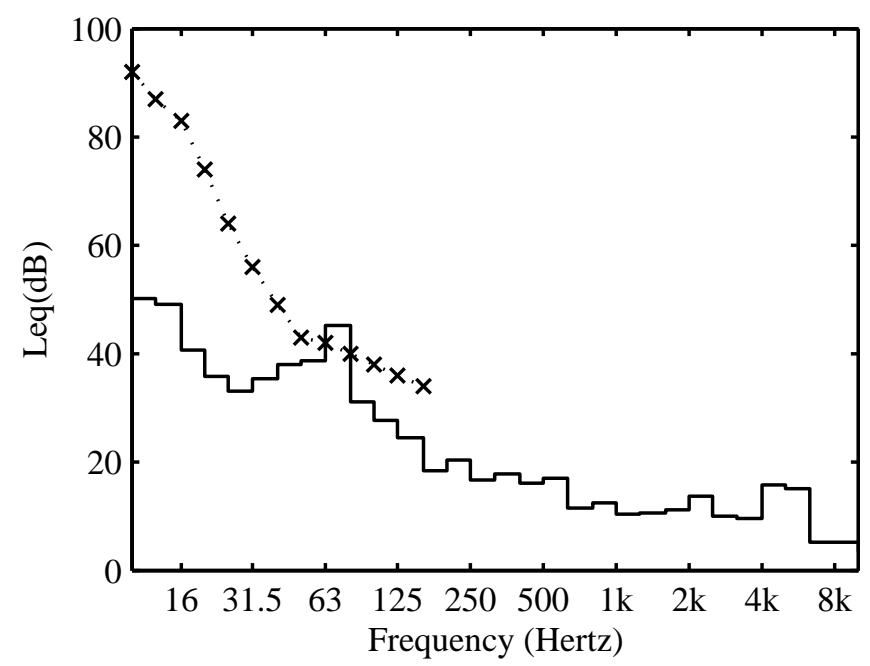




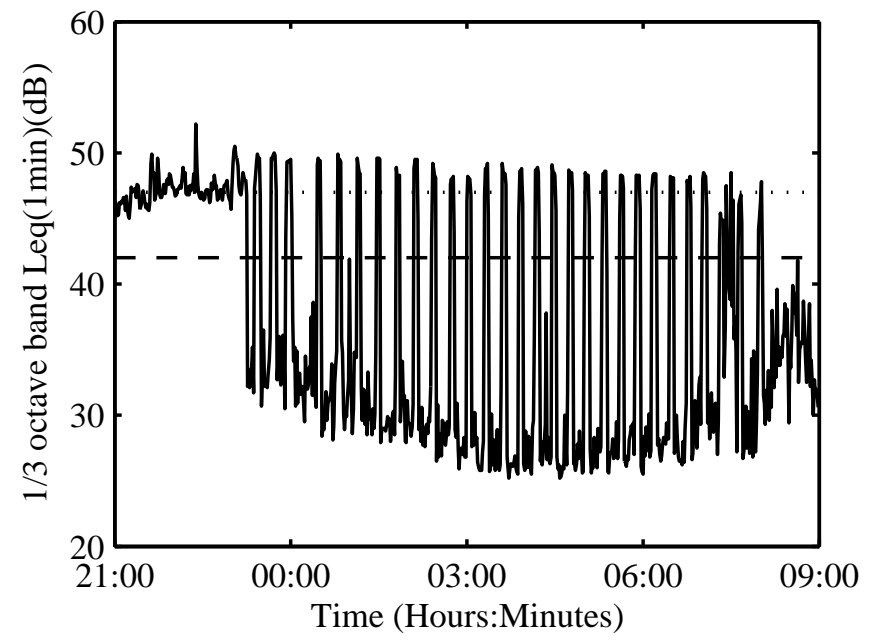




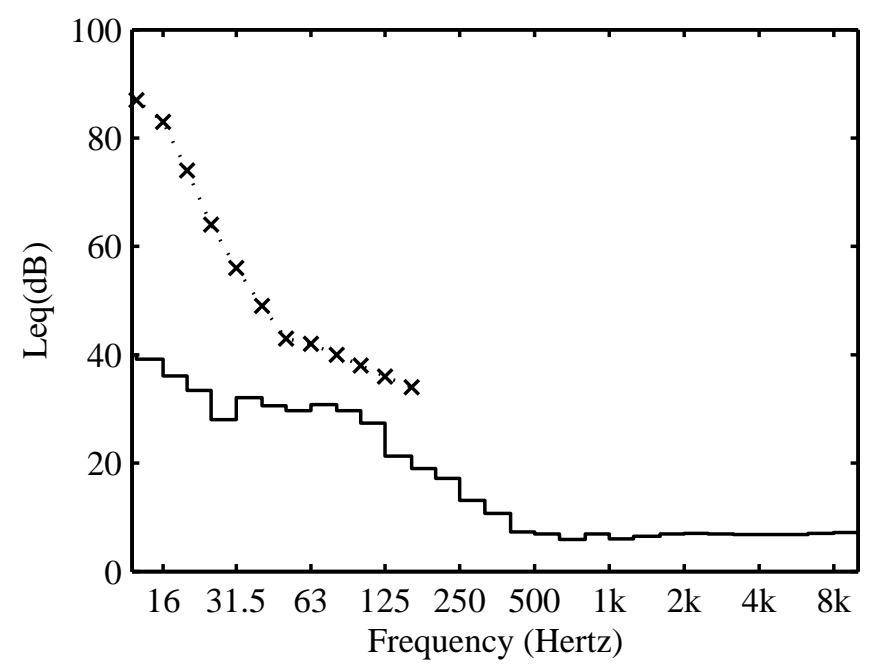




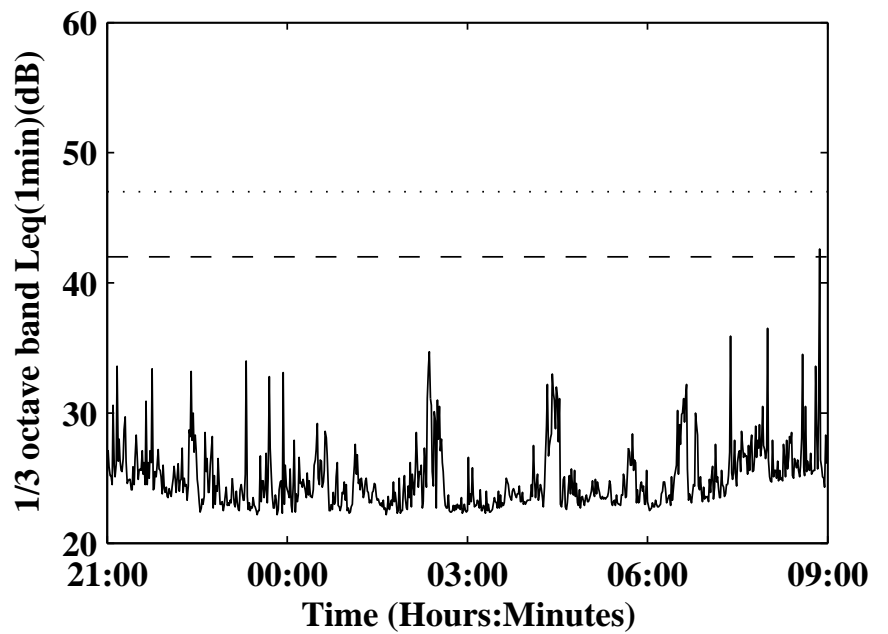




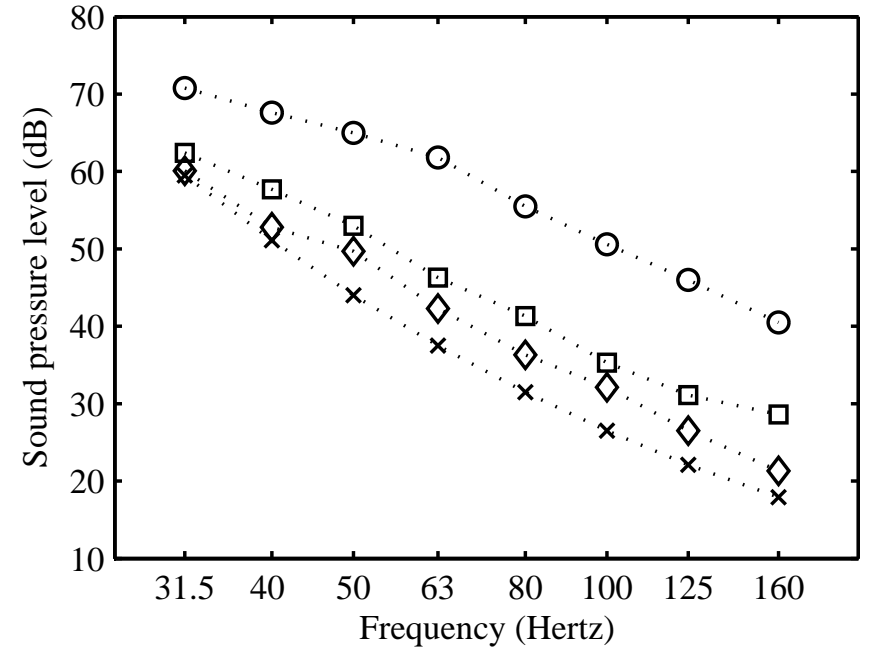




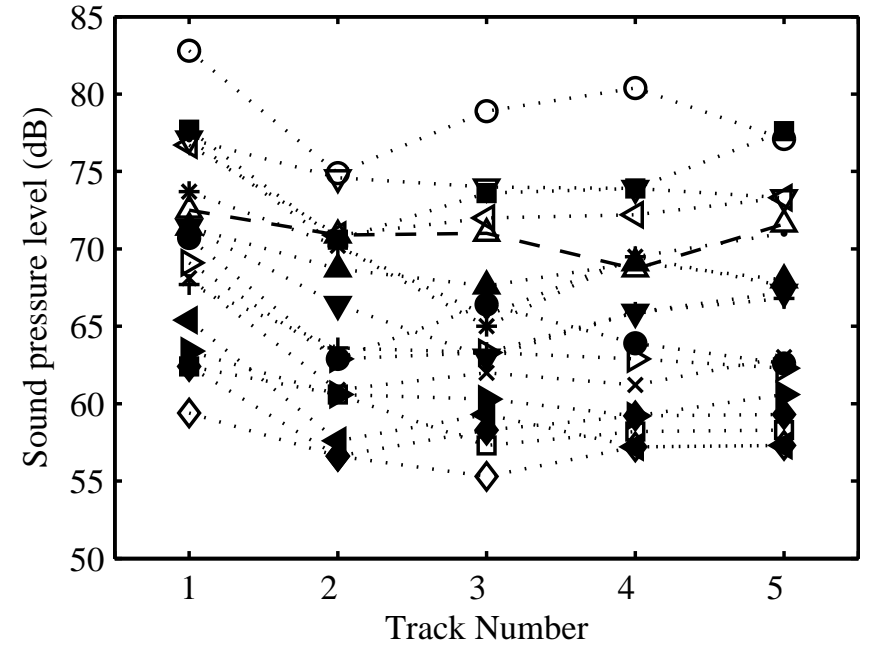




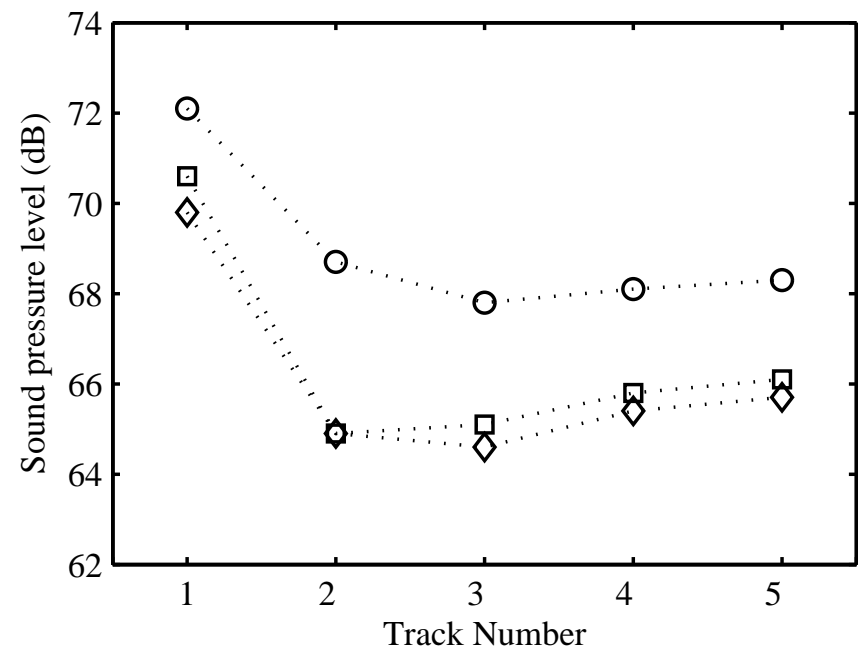




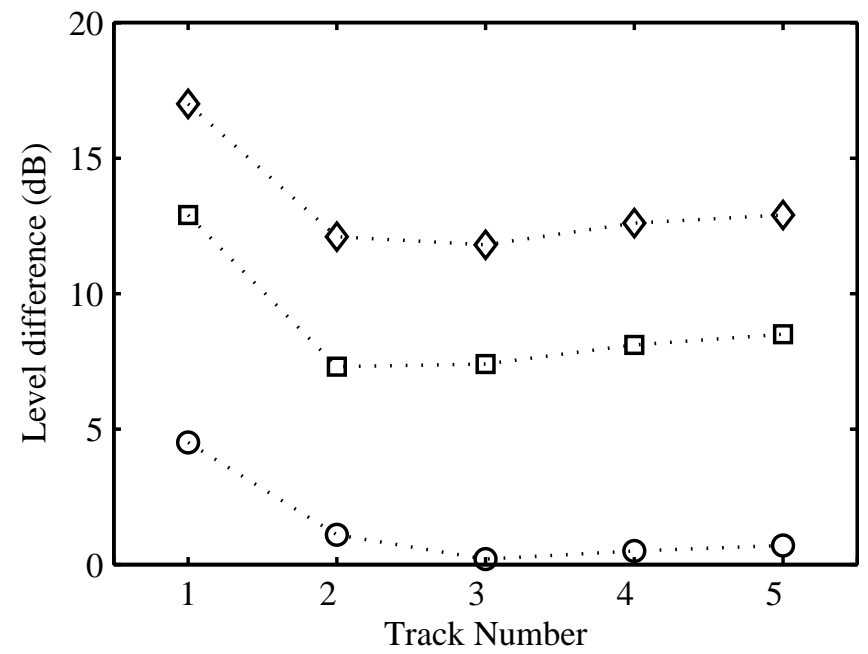




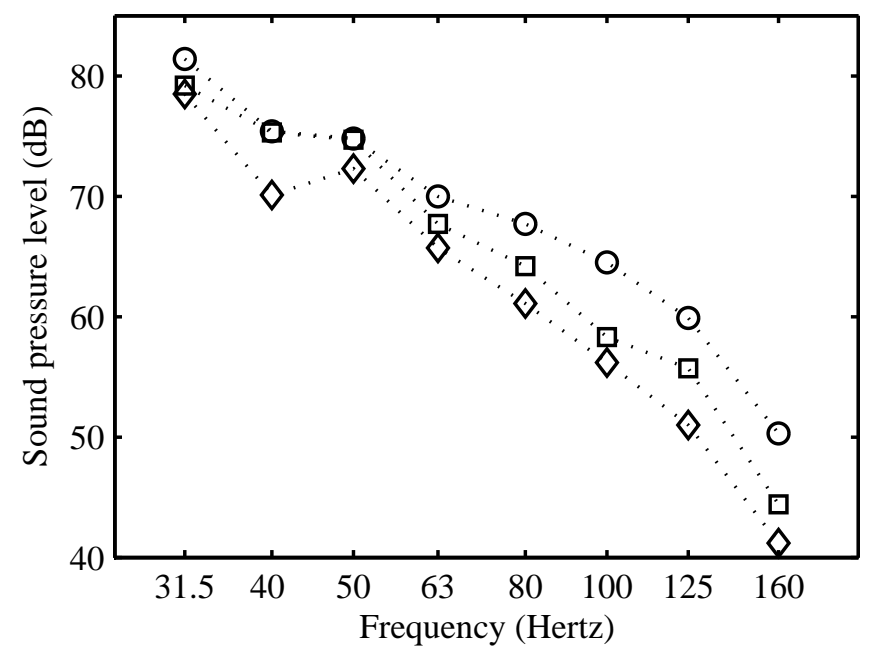




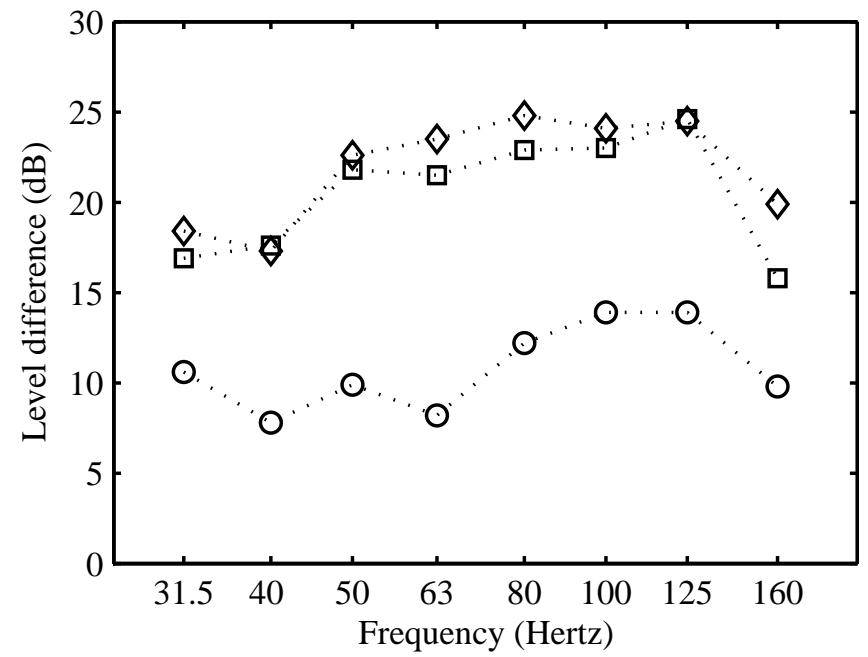




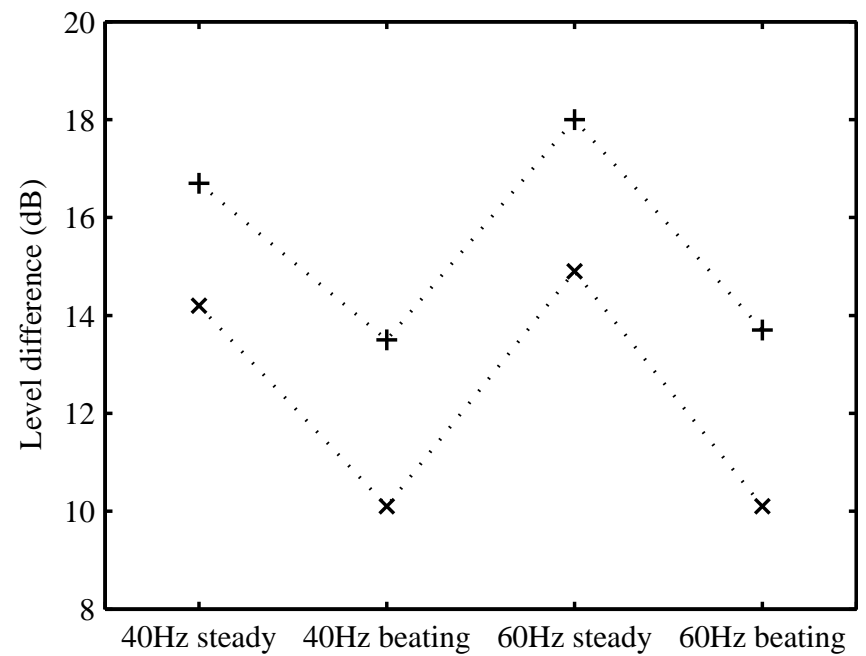




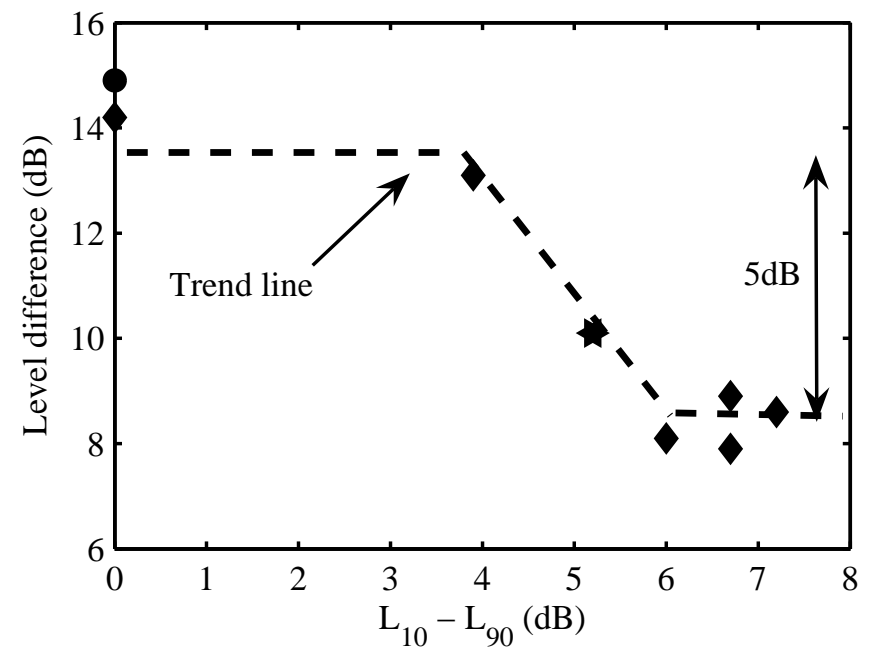




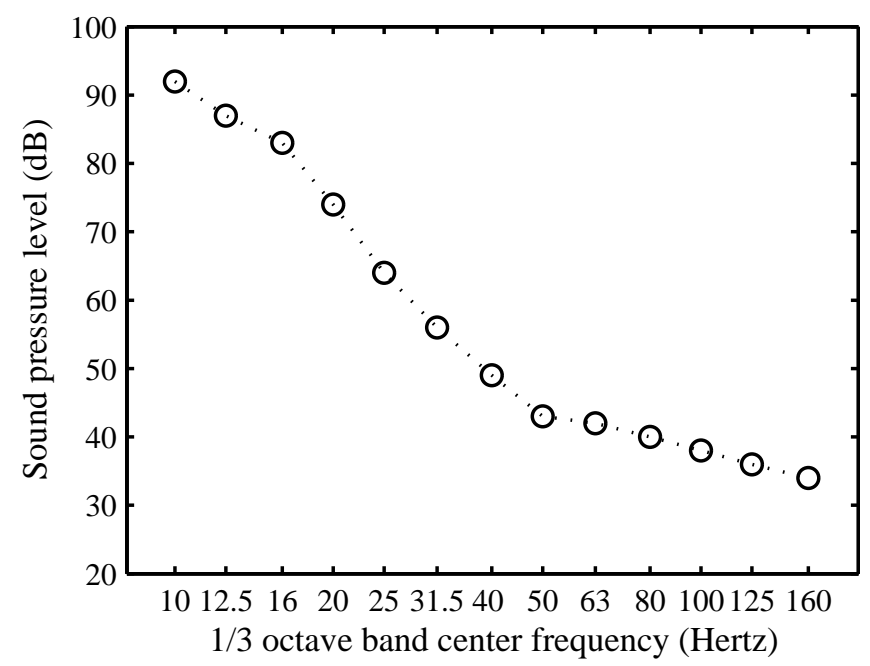

